\title{
Neisseira meningitidis Serogroup W causing primary meningococcal septic arthritis in a toddler and review of the literature
}

\section{L.Corominas MD $\mathrm{PhD}^{1 *}$, I.Manent $\mathrm{MD}^{2}$, E.Velasco $\mathrm{MD}^{3}$ and A.Peiro $\mathrm{MD}^{1}$}

${ }^{1}$ Pediatric Orthopedic Surgeon .Hospital Sant Joan de Deu. Barcelona

${ }^{2}$ Pediatric orthopedic resident. Fundació hospital Esperit Sant

${ }^{3}$ Pediatric medical doctor. Hospital Sant Joan de Deu. Barcelona

\author{
"Correspondence author \\ Laura Corominas \\ Hospital Sant Joan de Deu \\ Passeig de Sant joan De Deu 2 \\ 08950 Esplugues del Llobregat \\ Barcelona \\ Spain
}

Submitted : 08 April 2020 ; Published : 28 April 2020

\begin{abstract}
Since 2009, there has been a serious increase in the number of meningococcal infections in Europe caused by the serotype $W$ meningococcus (MenW:cc11). Invasive meningococcal disease is typically known to cause severe sepsis or meningitiswith high overall mortality rates of $10 \%$ despite adequate antimicrobial treatment. Focal infections such as arthritis incidentally occur as a complication of systemic disease.
\end{abstract}

Method We describe a rare case of primary meningococcal W135 arthritis in a 2-year-old patient.

Results Arthrocentesis of the right Ankle while patient was under anesthesia, yielded grossly purulent fluid, so we made arthrotomy and drainage. The culture from synovial fluid revealed $N$. meningitidis. The status of the patient improved after surgical drainage and intravenous antibiotic therapy. She recovered completely after 1 month.

Discussion.Atypical clinical presentation is associated with higher case fatality rates and can lead to misdiagnoses.an unusual presentation of invasive meningococcal infection and the early identification of the bacteria, combined with the correct treatment, prevent the complications and even death.

Keywords: Serogroup W; primary meningococcal septic arthritis; toddler; atypical presentation

\section{Abbreviations:}

1. Serogroup $\mathrm{W}$ meningococci ( MenW),

2. Invasive meningococcal disease ( IMD)

3. Primary meningococcal arthritis (PMA)

4. C-reactive protein (CRP)

5. Erythrocyte sedimentation rate (ESR)

6. Primary meningococcal septic arthritis (PMSA)

\section{Introduction}

Neisseria Meningitidis is associated with severe invasive infections such as meningitis and fulminant septicemia among others. Musculoskeletal infections associated with N. meningitidis have been reported exceptionally.

Septic arthritis secondary to meningococcemia has been reported in $10 \%$ cases Serogroup W meningococci (MenW), also referred as serogroup W-135 menigococci, and was first identified in the 1960s [1, 2]. In the following years, MenW ST-11 cases remained low at endemic levels through most of the early 2000s. However, the prevalence of meningococcemia caused by MenW have been increasing in Europe, Africa, North America and the Middle East3. Men W ST11 has been found associated with different complications including pericarditis, peritonitis, acute GI symptoms (vomiting, diarrhea and nausea), and severe respiratory tract infections and in rare cases, septic arthritis. Although most of these presentations and symptoms are described in all the Invasive meningococcal disease (IMD), its prevalence has always remained low and its presentation as presenting sign has been rarely reported [3, 4]. Since 2009, there has been a serious increase in the number of meningococcal infections in Europe caused by serotype W 
meningococcus (MenW: cc11) [5].

Higher fatality rates have been observed in cases with atypical presentations where diagnosis may be delayed, Such risk highlights the need to consider IMD in the differential diagnoses when acute GI symptoms, septic arthritis or bacteremia pneumonia are present, primarily in regions where serogroup $\mathrm{W}$ is prevalent, We report a rare clinical case of a 2-year old girl with primary meningococcal arthritis (PMA) without meningococcemia [5-7].

\section{Case Presentation}

A 2-year old girl was referred to the emergency department of a third level pediatric hospital with a 24hours history of fever, progressive pain and swelling of her right ankle. There was no previous history of overseas travel, contact with animals or trauma. She had received only two doses of meningitis type $\mathrm{C}$ vaccine, according to the current immunization schedule in Spain in 2019.

On physical examination, right ankle was swollen, range of motion decreased and weight bearing was impossible due to severe pain. Her body temperature was $39.8^{\circ} \mathrm{C}$. No skin rash was observed and temperature measured at the ER was $39.8^{\circ} \mathrm{C}$. It is noteworthy to mention that no neck rigidity or photophobia was present at the moment.

The blood works showed a white cells count of $15^{*} 10^{\wedge} 3$ with $75 \%$ Neutrophils, C-reactive protein $(\mathrm{CRP})=139 \mathrm{mg} / \mathrm{l}$, normal $<15 \mathrm{mg} / \mathrm{l}$, erythrocyte sedimentation rate (ESR) $15 \mathrm{~mm} / \mathrm{h}$, normal $<1 \mathrm{~mm} / \mathrm{h}$, procalcitonin $(\mathrm{PCT})=8,63 \mathrm{ng} / \mathrm{m}$, normal $<0.5 \mathrm{ng} / \mathrm{m}$. A blood test culture was taken.

Upon clinical suspicion of septic arthritis of the ankle due to 3 out of 4 points on the Kocher criteria, A right ankle aspiration was performed at the emergency department using an equimolar 50\% nitrous oxide/oxygen gas premix (Kalinox ${ }^{\circledR}$ ), obtaining purulent synovial fluid. Cytological evaluation of the synovial fluid showed countless leukocytes and low glucose. The polymerase chain reaction (PCR) was positive for Neisseria meningitidis serotype $\mathrm{W}$ and the meningococcus also was grown in the synovial fluid culture. Intravenous antibiotic therapy was started with ceftriaxone for 5 days, followed by oral amoxicillin for 2 weeks. Blood cultures were negative. Infection parameters in repetitive blood tests were normalized 2 weeks later, with follow-up for 6 months; she made a full recovery without sequeale. Attending health workers who attended the patient and the parents were treated with ciprofloxacin $750 \mathrm{mg}$ monodoses as prophylaxis and Pediatric relatives with rifampicin.

\section{Discussion}

Neisseira Meningitidis is a pathogenic Gram-negative intracellular diplococcus. Transmission of the bacteria occurs through direct contact with respiratory droplets [5]. Thirteen distinct serogroups have been identified but invasive meningococcal disease is only caused by serotypes A, B, C, W and $\mathrm{Y}$. In young adults, a prevalence of approximately $24 \%$ has been reported [8]. Notwithstanding, incidence in older adults and infants is lower (5-8\%) [9].

In 2001, routine conjugate Meningococcal-C immunization was implemented in the immunization schedule in Spain during the first 2-4-6 months of life, and repeated at the age of 10. However, a dramatic increase of invasive meningococcal disease from a clonal complex variant of serogroup W (cc11) has been observed in our country during the last decade, which forced our health system to modify the 2020 immunization schedule. This leads to replace the Men- $\mathrm{C}$ vaccine by the Men -ACWY vaccine in our immunization schedule against meningococci A, C, W and Y, with a scheme of one dose at 4 months of MenC-TT and one dose of Men ACWY at 12 months and another at 12-14 years of age PMA is an uncommon form of meningococcal disease, defined as acute septic arthritis without meningitis or classical syndrome of meningococcemia, defined as the combination fever, rash and hemodynamic instability.

Reports of meningococcal disease presenting as septic arthritis date back to the 1980s, when Brawley et al reported a 22-month-old arriving at the hospital with a swollen and painful knee [10]. Following diagnosis of acute septic arthritis, the infectious agent was identified as MenW. The patient showed no rash or classic sign of meningococcemia, giving the clinician no reason to suspect meningococcal infection, what is causing these atypical presentations? Several theories have been proposed to explain its pathophysiology, including mesenteric hypoperfusion, septic epiploic micro infarctions and immune complex deposition. It is well known that changes in virulence factors of a strain can induce a stronger inflammatory response [3]. In this case, the Polysaccharide $\mathrm{W}$ makes up the capsule of Men $\mathrm{W}$ generating a low immune response, making its clearance from the host difficult as a consequence. This may be the reason why Men W ST11 strains have been associated with unusual primary infections sites.

Previous studies have suggested a higher virulence of W-135 compared to other strains [11]. It is important to consider that polysaccharide $\mathrm{W}-135$ has a reduced capacity to generate immune response, hindering the elimination of the infection in the host. The relevance of this agent is reflected in an increased fatality rate of meningococcal disease since its appearance: death rate has been reported for serogroup B around 10-12\%, while a higher death rate $(25 \%)$ for $\mathrm{W}-135$ has been observed. With the recent rise in global W-135 and the massification of the quadrivalent vaccine, we must be aware on how this will affect the incidence of meningococcal septic arthritis.

Septic arthritis by meningococcus is uncommon. According to published records, joint disease by meningococcus would occur in up to $10 \%$ of meningococcal diseases and can manifest itself in three types according to its presentation [12]. The first type presents at the beginning with positive cultures in synovial fluid. This is called primary meningococcal arthritis and is a very rare form of presentation. The second form is a reactive 
arthritis presented on average 10 days after starting treatment for meningococcal disease. The third type is a haemarthrosis by coagulopathy.

Localized infections most often occur as a complication of systemic disease, whereas primary localized infections (pneumonia, epiglottitis, endophtalmitis, pericarditis and arthritis), as in our patient, are rare. Arthritic involvement resulting from direct haematogenous spreading of circulating bacteria is not an uncommon feature of invasive meningococcal disease, in particular for serotype W. The pathophysiology of primary meningococcal septic arthritis (PMSA) involves an acute transient bloodstream infection with a subsequent invasion of the synoiva. $N$ Meningitidis can be isolated from synovial fluid while signs of meningitis or septicemia are absent.

PMSA is more frequently monoarthritic, affecting either the knee or ankle joint. It occurs in $<3 \%$ of meninogococcal infections [13]. As previously mentioned, PMSA by serogroup W135 is extremely rare. To the best of our knowledge, only 5 cases have been described in the pediatric literature, 4 cases involved a monoartrithis of the hip and 1 case of the knee [10, 14-16]. The case we report is the first known case of primary monoarthritis of the ankle due to Neisseria meningitidis $\mathrm{W}-135$. In an immunocompetent child with acute ankle pain and swelling as the only symptom upon presentation at the hospital. The case reported reflects that Meningococcal infection must be considered in the diagnosis of any child presenting with arthritis, even if afebrile and without rash.

The definite diagnosis of PMSA in our patient was based on positive synovial fluid cultures without clinical signs of meningitis or the classical syndrome of meningococcemia defined by the combination of fever, rash and hemodynamic instability.

\section{No external funding for this manuscript.}

Financial Disclosure: Authors have indicated they have no financial relationships relevant to this article to disclose.

Conflict of Interest: Authors have indicated they have no potential conflicts of interest to disclose.

Short Title: Neisseira Meningitidis caused septic arthritis in a toddler

\section{Conclusion}

It is important that the physicians become more aware of unusual presentations of meningococcal disease such as PMSA. With prompt diagnosis and initiation of treatment, the prognosis is excellent.

\section{References}

1. Kappan SL, Schutze GE, Leake JA, William J Barson, Natasha B Halasa, et al. (2006) Multicenter surveillance of invasive meningoccal infections inchildren. Pediatrics 118: e979-e984.
2. Kelly D and Pollard AJ (2003) W135 in Africa:origins, problems and perspectives. Travel medicine and Infectious disease 1: 19-28.

3. Stinson C, Burman C, Presa J, Abalos M (2002) Atypical presentation of invasive meningococcal disease caused by serogroup $\mathrm{W}$ meningococci. Epidemiology and infection 148: e12; 1-8.

4. Giamarellos-Bourboulis EJ, Greck P, Toskas A (2002) Primary meningococcal arthritis :case report and clinical review. Clin Exp Rheumatol 2002: 553-554.

5. Fidder AR, de Hartog B, faber T (2019) BMJ case rep 12: e229510.

6. Knol MJ, Susan JM Hahne, Jay Lucidarme, Helen Campbell, Hester E de Melker, et al. (2017) Temporal associations between national outbreaks of meningococcal serogroup $\mathrm{W}$ and $\mathrm{C}$ disease in the Netherlands and England: an observational cohort study. Lancet Public Health 2: e473-e482.

7. Mori N, Tomofumi Hayashi, Hideki Nakamura, Hideyuki Takahashi (2018) Meningococcal meningitis with neurological complications and meningoccemia due to serogroup $\mathrm{W}$ sequence type 11 complex. Journal of infection and Chemotherapy 24: 398-400.

8. Harrison LH, Trotter CL, Ramsay ME (2009) Global Epidemiology of meningococcal disease. Vaccine 27: B51-B63.

9. Christensen H, May M, Bowen L, Matthew Hickman, Caroline L Trotter (2010) Meningococcal carriage by age: a systematic review and meta-analysis. Lancet Infect Dis 10: 853-861.

10. Brawley RI (1980) Acute Septic arthritis caused by Neisseria Meningitidis serogroup W -135. Southern Medical Journal 73: 395-396.

11. Moll-Manzur C, Faundez F, Araos-Baeriswyl E, Monsalve X (2016) Artritis septica de rodilla por Neisseria Meningitidis serogrupo W-135: primer caso reportado en un adulto. Med Clin (Barc) 147: 225-226.

12. Gee C, Tandon T, Avasthi A, Jerwood S, Rao BM, et al. (2014) Primary Meningococcal septica arthritis of the ankle joint: a case report. J Foot ankle Surg 53: 216-218.

13. Ricci S, Montemaggi A, Nieddu F, D Serranti, G Indolfi, et al. (2018) Is primary meningococcal arthritis in children more frequent than we expect? Two pediatric case reports revealed by molecular test. BMC infect Dis 18: 703 .

14. Bilavasky E, Yarden-Bilavasky H, Zevit N, Jacob Amir (2006) Primary meningococcal artritis in a child: case report and literatura reivew. Scand j infect dis 38: 396399.

15. McMullan B (2009) An infant with meningococcal artrhtis of the hip. J Paediatr Child Health 45: 762-763.

16. Apfalter P, Hörler R, Nehrer S (2000) Neisseria Meningitidis serogroup W-135 primary monoarthritis of the hip in an immunocompetent child. Eur J Clin Microbiol Infect Dis 19: 475-476.

Copyright: (C2020 Laura Corominas. This is an open-access article distributed under the terms of the Creative Commons Attribution License, which permits unrestricted use, distribution, and reproduction in any medium, provided the original author and source are credited. 